\title{
An Uncertainty Quantification Framework for Autonomous System Tracking and Health Monitoring
}

\author{
Matteo Corbetta ${ }^{1}$, Chetan S. Kulkarni ${ }^{2}$, Portia Banerjee ${ }^{3}$, and Elinirina I. Robinson ${ }^{4}$ \\ 1,2,3,4 KBR Inc., NASA Ames Research Center, Moffett Field, 94035 CA \\ matteo.corbetta@nasa.gov \\ chetan.s.kulkarni@nasa.gov \\ portia.banerjee@nasa.gov \\ elinirina.robinson@nasa.gov
}

\begin{abstract}
This work proposes a perspective towards establishing a framework for uncertainty quantification of autonomous system tracking and health monitoring. The approach leverages the use of a predictive process structure, which maps uncertainty sources and their interaction according to the quantity of interest and the goal of the predictive estimation. It is systematic and uses basic elements that are system agnostic, and therefore needs to be tailored according to the specificity of the application. This work is motivated by the interest in low-altitude unmanned aerial vehicle operations, where awareness of vehicle and airspace state becomes more relevant as the density of autonomous operations grows rapidly. Predicted scenarios in the area of small vehicle operations and urban air mobility have no precedent, and holistic frameworks to perform prognostics and health management (PHM) at the system- and airspace-level are missing formal approaches to account for uncertainty. At the end of the paper, two case studies demonstrate implementation framework of trajectory tracking and health diagnosis for a small unmanned aerial vehicle.
\end{abstract}

\section{INTRODUCTION}

The application of systematic methodologies for quantification of uncertainty in the area of system autonomy is a necessity to enable safe and efficient operations, diagnosis, and system recovery in case of partial failures. This need is driven by multiple factors. First, increasing levels of autonomy require holistic state awareness capabilities; knowing the system condition and its dynamic performance is a need to assess whether its behavior will adhere to future steps of the mission. Relevant system states are typically hidden, and state awareness is achieved with limited sensor data from which unobservable variables are estimated. The process is charac-

\footnotetext{
$\overline{\text { Matteo Corbetta et }}$ al. This is an open-access article distributed under the terms of the Creative Commons Attribution 3.0 United States License, which permits unrestricted use, distribution, and reproduction in any medium, provided the original author and source are credited.

https://doi.org/10.36001/IJPHM.2021.v12i3.2936
}

terized by sensor performance (which generate uncertainty in the measured variables) and uncertainty caused by the problem formulation often involving a physics-based, empirical, or purely data-based model. Second, the system and its operation may be affected by exogenous variables that are hard to model, and therefore their quantification relies on loose approximations on what the system may encounter during its mission. The uncertainty introduced by such simplifications should be accounted for when performing look ahead forecasts. Then, predictions are needed to compute indicators suggesting, directly or indirectly, the probability to accomplish the mission. The computation depends on a set of assumptions related to: system state and performance, observed degradation, computational models used to simulate/represent the system behavior, and external variables affecting system operations and their corresponding future values.

As previously demonstrated in the PHM domain, predictions should always be accompanied by confidence intervals (Saxena et al., 2008; Sankararaman, 2015). All the hypotheses on models, external variables and observations lead to estimates that will not replicate the exact future behavior, but they will represent it in a simplified fashion. Therefore, prognoses founded on computer models cannot be naively interpreted as deterministic outcomes, because of the several, simplifying assumptions they are based upon. The analysis proposed here, which looks at system tracking and health management with holistic perspectives, suggests a framework to list the possible sources of uncertainty, discusses which sources (through quantitative or qualitative approaches) should be modeled within the problem formulation, and defines the challenges of uncertainty-aware predictive estimation for autonomous or automated systems.

Uncertainty quantification is already being investigated in a number of scientific areas, especially computer models. The literature on such topics is extensive, and examples include the work in (Najm, 2009) on uncertainty quantification for 
computational fluid dynamics, and in (Eldred \& Burkardt, 2009) on intrusive and non-intrusive spectral methods. In the case of system tracking and health monitoring tools, a number of uncertainty sources may enter the prediction problem (Sankararaman, 2015). When moving beyond componentlevel, efforts towards uncertainty management for systemlevel PHM are missing, lacking a framework to approach the design of the monitoring system systematically. Many engineering studies and applications list the source of uncertainty, (typically naming model, measure, and external variables), but it is unclear how those uncertainty are linked in the prediction process.

Further motivations for this work, which started by addressing uncertainty quantification and management methods of future low-altitude airspace operations, is discussed in the next subsection. The contribution of this paper and how it relates with existing works is addressed in Section 2 . The uncertainty framework is then split into sources and predictive process, Section 3. Section 4 shows two applications related to the airspace domain: (i) uncertainty affecting the tracking of a small unmanned aerial vehicle over a pre-defined route, and (ii) uncertainty affecting the design of a modelbased electric powertrain health monitoring system composed of Lithium-ion battery, electronic speed controller (ESC), and brushless DC motor.

\subsection{Motivation driven by increasing operations of auto- nomous aerial vehicles}

The number of unmanned aerial vehicles (UAVs) entering the low-altitude airspace is expected to increase drastically in the next decade (Kopardekar et al., 2016; FAA, 2018). This forecast, driven by current interests in autonomous or automated UAV operations like package delivery, surveillance, agriculture optimization, as well as future urban air mobility, suggests the need of systematic approaches to enable autonomous UAV operations efficiently and safely. Such a need is motivated by multiple factors. As addressed in (Kopardekar et al., 2016), unmanned systems will enter areas originally used by traditional, manned aviation. However, infrastructure and integration requirements were not originally developed to accommodate a mix of different vehicles and systems. Small, low-cost UAVs, which are likely to be utilized for package delivery and other operations, do not guarantee high reliability standards (King, Bertapelle, \& Moses, 2005; Freeman \& Balas, 2014; Johry \& Kapoor, 2016), suggesting reliability issues leading to high failure rates, when compared to commercial aviation. In the case of unmanned urban air mobility, the absence of an onboard pilot will require advanced stateawareness tools to ensure safety for passengers as well as on the ground, minimize failure rates and reduce service disruption.

In order to make autonomous and automated systems able to meet high safety requirements, taking the different uncertainty sources into account is crucial to the decision making process. However, the uncertainty sources and their quantification will vary depending on the level of autonomy of the considered system. While many definitions for autonomous systems have been proposed (Stevens, Lewis, \& Johnson, 2015), we chose here to adopt the taxonomy proposed by (Fong, 2018), which defines an autonomous system as a system capable of self-directedness to achieve goals, and self-sufficiency to operate independently. These two properties, self-directedness and self-sufficiency, highlight the difference with an automated system, that works with predefined instructions and commands such as a pre-planned trajectory. In other words, while operating, the automated system capabilities is limited to the command and control that were previously implemented, whereas the autonomous system is able to take its own decisions according to the situation and change the outcome of the mission. In this paper, the UAV path tracking problem reflects the operation of an automated system, that is a UAV operating on a pre-defined set of instructions. However, the approach is still applicable to different levels of autonomy, including autonomous systems as it was defined previously. That is the reason why this paper often refers to both autonomous and automated systems.

The effect of growing interest in low-altitude operations is a number of research activities on automated and autonomous UAVs, including new design, reliability, efficiency, and autonomous functions. Examples of such works can be found in (Hoffmann, Huang, Waslander, \& Tomlin, 2007) for flight dynamics and control, (Langelaan, Alley, \& Neidhoefer, 2011; Glasheen, Pinto, Steiner, \& Frew, 2019) for wind field estimation, (Krish nakumar et al., 2017) for safety of low-altitude UAVs, and (Balaban et al., 2017) for dynamic routing and decision making. More generally, interests in autonomous vehicles also generated a number of system-level research on the safety of the national airspace, as in (Liu \& Goebel, 2018). Some system health management and PHM concepts for UAVs were discussed in (Jing \& Haifeng, 2013; Walker, 2010), while an early study on fault detection for unmanned vehicles was presented in (Drozeski, Saha, \& Vachtsevanos, 2005).

\section{CONTRibution AND RELATED WORK}

This work proposes an approach to identify the sources of uncertainty and map them into what is defined as predictive process structure, inspired by concepts in (Smith, 2013). That notion is developed further to discuss how the uncertainty sources could be mapped within the predictive structure to ensure that the link among different elements (and their corresponding uncertainty) are captured. The critical analysis of uncertainty in prognostics and remaining useful life prediction in (Sankararaman, 2015) highlights that the classical distinction between aleatory and epistemic uncertainty may 
not be as effective when dealing with far-ahead predictions, which is the theory our work agrees with and incorporates in the framework. The definition of uncertainty sources as aleatory or epistemic best fit computer models and their verification and validation, rather than future predictions. However, some uncertainty sources in the framework still benefit from such a distinction, and it will be used when believed to be appropriate. Differently from (Sankararaman, 2015), this paper focuses more on the type of uncertainty source, and their mapping in a predictive process, rather than the significance and interpretation of uncertainty in remaining useful life estimations. This work is a direct extension of (Corbetta \& Kulkarni, 2019), where initial concept of the framework is proposed. This research also leveraged material from (Roy \& Oberkampf, 2011), and previous works on uncertainty for PHM applications presented in (Sankararaman, Ling, Shantz, \& Mahadevan, 2009; Sankararaman \& Goebel, 2015; Sankararaman, 2015; Goebel, 2017). The concepts developed in those works have been helpful to define the proposed framework, so the reader may find a correlation between this manuscript and those earlier works. Details on verification and validation of computer models with associated uncertainty does not constitute the goal of this paper, and the interested reader is referred to (Roy \& Oberkampf, 2011) for details. Focusing on the framework level, it is worth mentioning the remarkable result of SANDIA National Laboratory with DAKOTA, a software tool for uncertainty quantification (Adams et al., 2009).

Within the unmanned aerial vehicle domain, besides studies of uncertainty affecting aircraft routing (Jun \& D' Andrea, 2003) and methods for collision avoidance (Alba ker \& Rahim, 2009), methodologies to handle and approach uncertainty affecting UAV systems and operations has been rarely explored. The work in (Sankararaman, 2017) identified uncertainty sources influencing UAV operations, and presented examples of decision making strategies based on those uncertainty sources.

Specific statistical techniques used to quantify the uncertainty in computer models, sensors, or environmental variables are not discussed. The interested reader may refer to: (Saltelli, Tarantola, Campolongo, \& Ratto, 2004; Cacuci, 2003; Saltelli et al., 2008; Crestaux, Ma1^tre, \& Martinez, 2009) for Sobol's indices and sensitivity analysis, (Ghanem \& Spanos, 1991) for spectral methods in finite element simulations, (Najm, 2009; Crestaux et al., 2009) for polynomial chaos expansion, and (Tessem, 1992) for interval analysis. For a broader overview of uncertainty quantification methods see (Smith, 2013) and references therein. Also, Bayesian filters, which are one of state-of-the-art methods for model-based filtering of past and present uncertainty, are not discussed as the interested reader is referred to (Kalman, 1960; Gordon, Salmond, \& Smith, 1993; Arulampalam, Maskell, Gordon, \& Clapp, 2002; Chen, 2003; Haug, 2005), and references therein for a comprehensive overview.
The goal of this framework is to provide a systematic procedure to identify and quantify uncertainty, from the perspective of system-level PHM for autonomous or automated systems. This effort is different from the previous, cited work for the following reasons. It does not develop new statistical techniques, nor apply existing ones to new problems. Rather, it discusses the problem of uncertainty quantification for unmanned system tracking and health monitoring, highlighting key steps and challenges. A methodology is proposed to list, define, and map uncertainty in the predictive process structure. This work does not focus entirely on computer models, as done by a large number of existing studies. It also discusses the role of measurement, algorithms, and external forcing. The analysis shows the importance of predictive capabilities to assess multiple future scenarios based on those uncertainty sources, current available information and potential mission profiles.

The proposed methodology is system agnostic, so the framework can be applied to different systems with appropriate tuning. The case studies reported in Section 4 will discuss implementation of the framework and the selection of appropriate statistical techniques to handle specific challenges.

\section{UnCertainty Sources, QUantification, AND Sig- NIFICANCE}

This section has three main goals. First, it reviews the importance of uncertainty quantification involved in predictions of autonomous systems operations and health management. Then, it discusses a high-level subdivision of uncertainty, refined from the precursor of this work (Corbetta \& Kulkarni, 2019), which mainly combines information from Chapter 1 of (Smith, 2013), (Roy \& Oberkampf, 2011), (Goebel, 2017), and (Sankararaman, 2015), and other works that will be cited appropriately within the section. Then it defines a predictive process structure, where uncertainty propagates through the different elements composing the predictive process.

\subsection{Some remarks on uncertainty quantification for au- tonomous systems}

For the sake of this work, system tracking is defined as the continuous observation (or estimation from observable variables) of system's key parameters and telemetry variables to assess the adherence of the system to its mission. Key parameters include, for example, current position, kinematic profile, attitude and battery state of charge. When focusing on autonomous vehicles, the current position may not be enough to assess whether the vehicle is operating as planned. We include battery state of charge under the system tracking umbrella, since its value is necessary to evaluate if the vehicle can complete the mission; it is not necessarily a health parameter like, e.g., capacitance. Health monitoring can be assessed through either dedicated sensor data, like powertrain 
temperature, or a combination of health data and telemetry, like speed of motors.

Enabling predictive capabilities for automated or autonomous UAVs operations requires the analysis of more than conditionbased models and data only. It needs also to include operational input, which may change due to external factors, like other UAVs demanding right-of-way for emergency situations. However, that may not be enough to ensure safe operations. The third source of information composes of environmental variables that may affect UAV dynamics. This is particularly true for small UAVs expected to operate in urban environment, where wind tunnel effects and local gusts are hard to predict. However, wind is not the only variable affecting small UAV performance; temperature, besides being easier to predict, also plays a role in the integrity of the UAV electronic components.

Environmental conditions are particularly challenging for the rate and sparsity at which data can be usually collected, and some of them, like the wind field, require computationally intensive models even for the simplest look-ahead forecast. Also, autonomous vehicles may require an enhanced set of prediction capabilities in comparison to their automated counterparts. In the latter case, one may use models and historical sensor data to enable the prediction of environmental variables along pre-defined sets of options. The computing effort can be dedicated to those set of possibilities ignoring the rest of the environment. In the case of an automated UAV, the set of possible options is typically finite, and restricted to the previously defined instructions. An autonomous vehicle with self-directedness has a pool of options that may be, theoretically, infinite. Thus, autonomy may be affected by greater uncertainty than automation. Predictions for autonomous system behavior and operations require in-time assessment capabilities. For example, an autonomous UAV operating in a wind field should include expected wind gusts or mean wind profile along the possible routes. To do so, a wind forecast tool is necessary to evaluate multiple options explored within the decision making framework.

\subsection{Uncertainty Sources}

This section discusses macro-categories model, method, measure, and input that have been chosen to represent the uncertainty sources of predictive estimation ${ }^{1}$. Each of them comprises of sub-categories which are depicted in Figure 1.

\subsubsection{Model}

The sources of model uncertainty have been divided in model abstraction, model parameters, and model error. Model abstraction refers to the hypotheses introduced during model

\footnotetext{
${ }^{1}$ This section uses the following notation: bold, lower-case letters to define vectors and random vectors, e.g., $\boldsymbol{x}$, and functions and vector functions are defined by parentheses that highlight dependencies, e.g., $f(\cdot), \boldsymbol{f}(\cdot)$.
}

development with the intent of representing reality and physical processes through a set of equations. Those hypotheses include neglecting or simplifying (for example, through linearization or reduced order models) physical phenomena, environmental effects, and other external factors that may interact with the system, but their effect on the quantities of interest (QoIs) is believed to be limited.

Model parameters include fixed or variable coefficients required to estimate the output QoIs from the model. They may fall within a range of values, or defined by random variables and therefore represented through probability density functions (pdfs). They may depend on system's properties, and they may also evolve over time because of degradation phenomena. However, time-varying external forcing, like environmental variables, are not included within the parameter category. Those are considered as inputs and will be discussed in Subsection 3.2.4.

Model error introduces the uncertainty representing the difference between model and observed outcome, when other uncertainty sources (model abstraction and parameters) are already accounted for in the prediction. As a matter of fact, a rigorous discussion should distinguish between modeling errors and residuals, as the former represent the deviation of the model output from the true (unobservable) value of the QoI, and the latter defines the deviation between model output and the observed value of the QoI. For the goal of this work, we will not distinguish between the two, and the interested reader can refer to dedicated literature on statistical models and online material on the subject. By abusing the nomenclature, we will use residuals and errors interchangeably even if their definition is slightly different. The model error is typically an additive term whose properties depend on the difference between observations and model predictions. In the optimal case, the model error is a zero-mean Gaussian variable with constant variance, e.g., $\epsilon \sim \mathcal{N}\left(0, \sigma^{2}\right)$. For illustration, consider the following model:

$$
f_{\theta}(x, q)
$$

Vector $\boldsymbol{x}$ represents independent variables influencing the QoI or system state variables, while $\boldsymbol{q}$ represents the collection of external inputs. The latter is a (possibly nonlinear and multidimensional) mapping function $f(\cdot, \cdot)$ parameterized by the parameter vector $\boldsymbol{\theta}$. In this case, model uncertainty lies in the hypotheses and assumptions used to define $f(\cdot, \cdot)$, as well as in the value(s), distribution(s) or parameter of the distribution(s) that define $\boldsymbol{\theta}$. Uncertainty driven by model abstraction is quantified through model validation, which is omitted here for the sake of brevity, and the reader may refer to (Smith, 2013; Roy \& Oberkampf, 2011) for details on the subject. Unmodeled physical phenomena are typically represented with additive terms that modify the model response based on the input values (both $\boldsymbol{x}$ and $\boldsymbol{q}$ ); such additive terms 


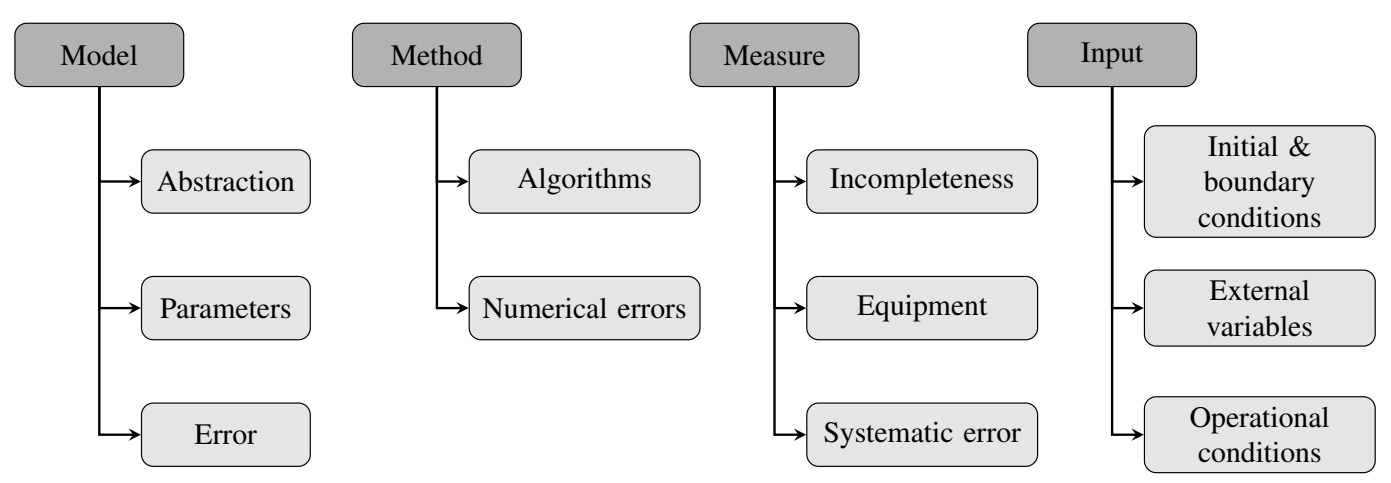

Figure 1. High-level classification of uncertainty sources encountered in the predictive process.

are meant to replace the "missing physics". By slightly modifying the notation in (Smith, 2013) (Eq. 1.5), the model equation becomes:

$$
\boldsymbol{f}_{\boldsymbol{\theta}}(\boldsymbol{x}, \boldsymbol{q})+\boldsymbol{\delta}(\boldsymbol{x}, \boldsymbol{q}) .
$$

Typically, the missing physics term is identified through datadriven methods, and so the form of the function $\delta(\cdot)$ and its parameters lose physical meaning. The model error can be quantified from the model fitting procedures after missing physics has been accounted for, and is typically additive. The model eventually assumes the following structure:

$$
\boldsymbol{f}_{\boldsymbol{\theta}}(\boldsymbol{x}, \boldsymbol{q})+\boldsymbol{\delta}(\boldsymbol{x}, \boldsymbol{q})+\epsilon .
$$

Some modeling choices, however, may drive the model error to be non-Gaussian and nonlinear, for example in damageprogression power laws.

\subsubsection{Methods}

Here, methods refers to the collection of algorithms and computation tools utilized to propagate information and variables through the system model, to compute the QoIs, or to interpolate or extrapolate variables from data. The sources of uncertainty belonging to this category have been divided into algorithms and numerical errors.

Algorithms for estimation, interpolation or extrapolation in multi-dimensional spaces introduce uncertainty because they may converge to sub-optimal solutions trying to minimize errors or loss functions. Such loss functions may be nonconvex, complex hyper-surfaces. As a consequence, different runs of the algorithms may generate different results because the algorithms remain trapped in local minima. This source of uncertainty is hard to quantify, since the initial conditions or initial guesses of the algorithm parameters or hyperparameters affect the solution, and because input data also change the loss function hyper-surface. Gradient-based optimization methods which depend on the initial guess of the system parameters and the input data are one example of algorithm source of uncertainty (for a practical example, consider the optimization of Gaussian process hyperparameters by maximizing the log-marginal likelihood, (Rasmussen \& Williams, 2006). Typical gradient-based minimization algorithms can generate different hyper-parameters at different runs depending on the initial guess). Gradient-free optimization methods dot not suffer, for obvious reasons, from the same issues related to initial-guess values as gradient-based methods. However, hyper-parameters as well as influence of the input data still play a role when estimating the final output.

Uncertainty does not lie only on the optimization of loss functions, but also in numerical errors raising by running computer models. Those include discretization, iterative, and round-off errors, as already discussed in (Roy \& Oberkampf, 2011). Discretization errors are caused by the finite grid used to compute the solution (e.g., finite element models). This is the case for atmospheric models when the numerical grid is larger than the scale of the physics that is being modeled (Smith, 2013).

Similarly to grid discretization, sampling-based methods like Monte Carlo and its variants (e.g., stratified sampling methods), suffer of uncertainty caused by the sample size, as already suggested in (Sankararaman, 2015). The right number of samples to solve multi-dimensional integrals may be prohibitive. In those cases the number of samples is reduced according to the computational power available, producing a coarse solution of the future state of the system or the time to reach a predefined bound (like in remaining life estimation problems). Such a solution might be affected by large uncertainty because the few samples are not capable of representing the true distribution shape, that can be multi-modal, heavy-tailed, etc.

Errors due to coding bugs are particularly hard to quantify, if not impossible, since the presence of a bug is unknown until it is discovered (and then fixed, so its contribution to uncertainty is then removed). As suggested in (Roy \& Oberkampf, 2011), numerical approximation errors should be explicitly represented by epistemic uncertainty, and sum together con- 
tributions assuming that the lower bounds of each error (discretization, iterative, and round-off) is zero.

\subsubsection{Measures}

This category includes measurement incompleteness, uncertainty caused by equipment, which translates into sensor calibration resolution, accuracy, and precision, and systematic errors generated by the measuring process, sensor installation and human error (if humans are involved). Measure incompleteness or missing measures are very common in complex measuring tasks, like estimating the GPS position of an object or collecting large amount of measures, like ocean surface water temperature (Luttinen \& Ilin, 2012). The most common (and optimistic) assumption for measurement systems is to be unbiased and Gaussian, which translates into: $\boldsymbol{y}=\boldsymbol{g}_{\boldsymbol{\nu}}(\boldsymbol{x})+\epsilon$, where $\epsilon \sim \mathcal{N}\left(0, \sigma^{2}\right)$, and $\boldsymbol{g}_{\boldsymbol{\nu}}(\cdot)$ is the measurement function parametrized by vector $\boldsymbol{\nu}$. This formulation is widespread in the field of state estimation, where the challenge is to infer the hidden state $\boldsymbol{x}$ from observations $\boldsymbol{y}$. Biases can be modeled as additive terms, i.e., $\boldsymbol{y}=\boldsymbol{g}_{\nu}(\boldsymbol{x})+\boldsymbol{b}+\epsilon$. It should be noticed that the uncertainty of the measurement noise, represented by variance $\sigma^{2}$, and the biases may depend on the input quantity, $\boldsymbol{b}=\boldsymbol{b}(\boldsymbol{x})$ and $\sigma^{2}=\sigma^{2}(x)$ (this last equation should be replicated for each element in $\boldsymbol{x}$, assuming uncorrelated measure errors). A wrong assumption on $\epsilon$ or $\boldsymbol{b}$ will affect the accuracy and precision of the hidden state estimation, even with a correct system model. It should be stressed that a similar model may be necessary to estimate input variables $\boldsymbol{q}$. Current input values to the system may aid the prediction of future inputs, thus refining the prediction problem and reduce uncertainty. Moreover, if a progressive degradation model is tied to the main system model, the estimate of input forcing may be necessary to estimate the severity of degradation growth.

Measurement accuracy may be hard to quantify in some realtime applications when on-demand calibration is not an option. Accuracy directly ties to biases, which may lead to incorrect inference over the latent variables. Precision is typically modeled by means of random variables, and indicates the dispersion of the observations that should not be attributed to the system but to the measurement device. The specificity of measurement uncertainty depends on instruments, methodologies, and constraints of specific applications, and so are not investigated any further here. More details on measurement uncertainty can be found in the ASME standard (Abernethy, Benedict, \& Dowdell, 1985). For an example of measurement model and corresponding uncertainty, the reader is referred to (Frew \& Sengupta, 2004) where the uncertainty of a $2 \mathrm{D}$ stereo-camera device was discussed to implement an obstacle avoidance methodology.

\subsubsection{Input}

The set of input includes time-dependent variables, initial and boundary conditions, and exogenous forces that may interact with the system and therefore affect its dynamics. Some inputs are actually operational requirements defined according to the system's intended function, and so they are called here operational input (e.g., desired trajectory of an automated or autonomous vehicle). External inputs are external forces or events which depend on the environment the system is operating into. Initial and boundary conditions are the most common exogenous inputs.

It should be noticed that other works may include input within the model category and discuss the corresponding uncertainty as part of model uncertainty. This is the case in two of the main sources we used as inspiration. First, (Roy \& Oberkampf, 2011) suggests that input includes "not only parameters used in the model of the system, but also data from the description of the surroundings, geometry, and initial conditions, ..." (Subsection 3.1). In (Smith, 2013), model input refers to the set of parameters "that must be specified before the model can be used to represent or predict the behavior of the process" (Chapter 1, Sub-subsection 1.1.2).

Differently from those works, here model parameters refer to system model coefficients (fixed or time-varying, as explained above), but the system input has a dedicated section, so there is a slight inconsistency between the categories we have provided when compared to the existing literature. The reason lies on the nature of the predictive process discussed in this paper, which differs from the goal of the aforementioned works concentrating mainly on computer models. By dividing model input and model parameters, we can distinguish between uncertainties coming from different, non-associated sources. Input refers to exogenous variables that do not belong to the system, but have a direct or indirect effect on it. The effect of a random wind field in an urban environment on a small UAV is a different input source than UAV properties like inertia moments, which are also input to the model. However, inertia moments are inherent system parameters depending on geometry and mass, do not change noticeably (apart from different vehicle configurations or damages happening to the airframe), and of course do not depend on the trajectory, differently from wind.

It should be noticed that the discussion in (Sankararaman, 2015) highlights that condition-based monitoring is not affected by "true variability", and therefore the distinction between aleatory versus epistemic uncertainty loses importance. On the other hand, when discussing input of automated or autonomous systems, external input are often characterized by both epistemic and aleatory components. The first is caused by measure errors, interpolation error or forecasts uncertainty, and the latter given by the intrinsic variability of wind phenomena. Uncertainty related to the health of the system is 
mainly epistemic, as already suggested in (Sankararaman, 2015), and can be reduced by collecting more data up to a point where measurement error is not affecting the predictive process performance. For those reasons, input and model parameters have been kept separate.

\subsection{Predictive Process Structure}

This section discusses some key aspects in mapping the uncertainty sources discussed in the previous Section into a predictive process. The predictive process is straightforward, and leans towards a generality rather than specificity. Figure 2 shows a diagram of the predictive process structure. It is similar to many existing frameworks utilized in PHM and other fields. The sources of uncertainty discussed in Figure 1 are tightly connected to this structure.

The difference spaces introduced in the predictive process are tightly connected to the sources discussed in the previous section. The measure space and the input space feed the models, which are collected into the representation space. The computing space embraces both methods and models, since algorithms (with their corresponding uncertainty sources discussed in the previous Section) may be required to solve both input and system models.

According to the goal of the tracking and health monitoring process, the predictive process can be simplified by removing some elements that do not apply to the goal, or whose uncertainty is expected to be limited and using a deterministic function or value does not affect the prediction (The latter should be supported by proper sensitivity analysis (Saltelli et al., 2008)).

\section{Application Case Studies}

In this paper, the approach to uncertainty quantification is demonstrated on an automated UAV flight comprising of trajectory tracking, and by modeling the UAV powertrain elements for health monitoring purposes.

In the first study, the UAV is commanded to fly through a set of pre-defined waypoints associated with their expected times-of-arrival (ETAs). The goal is to quantify the uncertainty of such ETAs as well as the uncertainty of the time of arrival along the whole path as the vehicle completes its mission. The quantification of such uncertainty can aid trajectory planning in high density airspace and flight scheduling. For example, one can compute the upper bound of the mission time for multiple UAVs and optimize fleet operations. Multiple sources are responsible for uncertainty in the time of arrival of the vehicle. First and foremost, external forcing like wind magnitude, direciton and turbulence intensity along the route. Without a high-fidelity model of the UAV, the autopilot response is also a source of uncertainty, together with vehicle dynamic properties and potential hinner degradataion of powertrain components. A comprehensive physics-based model to reduce uncertainty would require not only the vehicle high-fidelity model, but also high-resolution estimates of wind speed and direction on the flight route from field sensors and/or complex weather simulation models. The former (high-fidelity vehicle model) may actually be available at times, but typically there is a high cost associated with running the model multiple times. The latter (high-resolution wind field) is never available to UAV and fleet operators and is currently an open field of research. Thus, the assumption used for case study 1 is that the sources of uncertainty mentioned above contribute to variations of the flight cruise speed (that would otherwise be constant in-between way-points). That is a large simplification of the real phenomena, but constitute an appealing option when compared to the burden of high-fidelity simulations to be run at fast rate.

The second case study discusses what could be a potential model-based approach of UAV powertrain diagnosis, modeling three fundamental components, namely battery, ESC, and motor. For this specific example, variability of external forcing has been ignored. Similarly to the trajectory tracking case study, external forcing is trajectory dependent and depends on the wind field during flight. It is not agnostic to the UAV operation. For the sake of brevity, other uncertainty sources related to model abstraction and battery model parameters have been ignored or simplified, but are discussed in the dedicated section. The example aims at providing the guideline to the uncertainty that may be affecting the model as well as the diagnosis process based on that same model.

The first case study was developed in a Matlab environment without any ad-on Toolbox, while the second case study was developed in Python using packages NumPy, SciPy, and Matplotlib.

\subsection{Trajectory tracking}

Trajectory tracking is an important area of research in the field of flight guidance, navigation and control and has been extensively studied for manned aircrafts (Kaminer, Pascoal, Hallberg, \& Silvestre, 1998; Radmanesh, Kumar, \& Sarim, 2018). A few interesting studies pertaining to unmanned vehicles include (Sujit, Saripalli, \& Sousa, 2014; Davis \& Chakravorty, 2007) that presents flight planning algorithms to control a UAV under different wind conditions. Yet, uncertainty in predicted trajectory caused to speed variations has mostly remained unexplored for UAVs.

In our application, the framework for UAV trajectory tracking includes the trajectory generation algorithm to provide a kinematically-smooth trajectory. One method for generating smooth paths based on pre-defined waypoints $n+1$ waypoints $\boldsymbol{P}$ with their ETAs $\boldsymbol{t}_{\boldsymbol{a}}$ include the non-uniform rational Bspline algorithm, NURBS (Rogers, 2000). NURBS can compute piecewise-constant jerk polynomial curves thus avoid- 


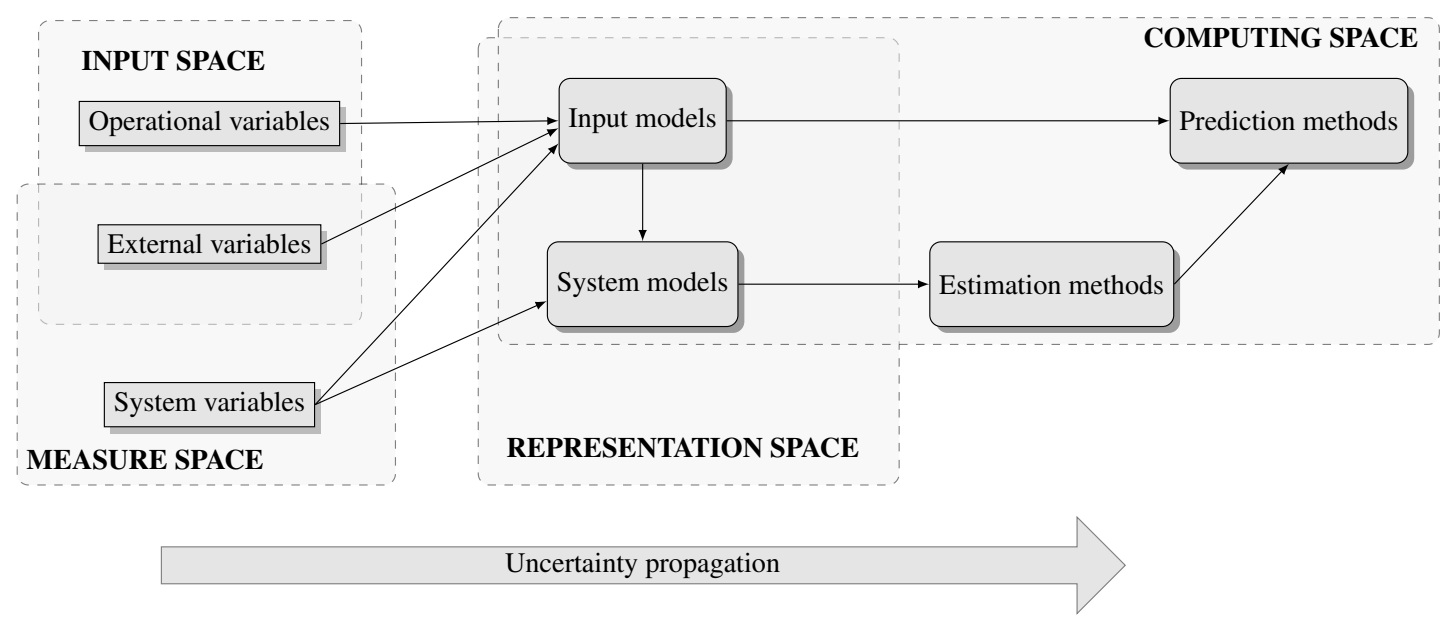

Figure 2. Predictive process structure. The bottom arrow describing uncertainty propagation does not indicate that uncertainty is introduced only in the measure and input spaces, but it rather indicates that all the elements in the predictive process introduce uncertainty, which increases from left to right.

ing discontinuity in the associated velocity and acceleration profiles, (Corbetta, Banerjee, Okolo, Gorospe, \& Luchinsky, 2019).

$$
P(u)=N U R B S\left(\boldsymbol{P}, \boldsymbol{t}_{\boldsymbol{a}}\right)
$$

Each segment of the NURBS curve is composed of a weighted contribution from each way-point defined according to a basis function $N_{i, k}(u)$ which is computed for $i^{t h}$ way-point and $k^{t h}$ degree, Eq. (2). The NURBS basis function is described in Eqs. (3a) and (3b).

$$
\begin{aligned}
x(u) & =\frac{\sum_{i=0}^{n} h_{i} N_{i, k}(u) x_{i}}{\sum_{i=0}^{n} h_{i} N_{i, k}(u)}, \quad 0 \leq u \leq n-k+2 \\
N_{i, k}(u) & =\frac{\left(u-t_{i}\right) N_{i, k-1}(u)}{t_{i+k-1}-t_{i}}+\frac{\left(t_{i+k}-u\right) N_{i+1, k-1}(u)}{t_{i+k}-t_{i+1}} \\
N_{i, 1}(u) & = \begin{cases}1 & \text { if } t_{i} \leq u \leq t_{i+1} \\
0 & \text { otherwise }\end{cases}
\end{aligned}
$$

The NURBS trajectory assumes that the UAV flies at constant velocity in between two consecutive waypoints. This is often not followed in real applications when external factors and environmental conditions introduces variations on the vehicle's expected speed producing uncertainty in the times of arrival (TAs) at all locations along the entire flight. If the uncertainty on the cruise speed is described by a Gaussian distribution, the uncertainty in the differential times-of-arrival can be computed according to error intervals (Physical Sciences 2, 2013) as denoted in Eqs. (4)- (5). Finally, the variance of the time of arrival at each way-point $k$ can be computed by cumulative sum of variances up to $\sigma_{t_{k}}^{2}$, as in Eq. (6).

$$
\begin{gathered}
\sigma_{t_{j, k}}^{2} \triangleq\left\{\begin{array}{ccc}
\Delta t_{a, k}{ }^{2} & \frac{\sigma_{v_{j, k}}^{2}}{\bar{v}_{j, k}^{2}} \quad \forall \quad \bar{v}_{j, k} \in \overline{\boldsymbol{v}}_{k}: \bar{v}_{j, k} \neq 0 \\
0 \quad & \text { otherwise }
\end{array}\right. \\
\sigma_{t_{k}}^{2} \triangleq \max _{j \in\{x, y, z\}}\left\{\sigma_{t_{j, k}}^{2}\right\} \\
\sigma_{t_{a, k}}^{2}=\sum_{i=1}^{k} \sigma_{t_{i}}^{2} .
\end{gathered}
$$

Eventually, the time of arrival at way-point $k$ can be described by a normal probability density function (pdf):

$$
t_{a, k} \sim \mathcal{N}\left(\bar{t}_{a, k}, \sigma_{t_{a, k}}^{2}\right)
$$

The confidence intervals of the position profile are hence computed by using the upper and lower intervals of the TAs,

$$
\begin{aligned}
& \mathcal{P}^{+}(t)=\operatorname{NURBS}\left(\boldsymbol{P}, \boldsymbol{t}_{a}^{+}\right) \\
& \mathcal{P}^{-}(t)=\operatorname{NURBS}\left(\boldsymbol{P}, \boldsymbol{t}_{a}^{-}\right)
\end{aligned}
$$

where,

$$
\begin{aligned}
\boldsymbol{t}_{a}^{+} & =\left[\bar{t}_{a, 0}, \bar{t}_{a, 1}+\sigma t_{a, 1}, \ldots, \bar{t}_{a, K}+\sigma t_{a, K}\right], \\
\boldsymbol{t}_{a}^{-} & =\left[\bar{t}_{a, 0}, \bar{t}_{a, 1}-\sigma t_{a, 1}, \ldots, \bar{t}_{a, K}-\sigma t_{a, K}\right] .
\end{aligned} .
$$

The NURBS trajectory along with the uncertainty bounds for an experimental UAV flight at NASA Langley Research Center are demonstrated in Fig. 3. The UAV is a DJIS1000 octocopter flying through a pre-defined set of 17 waypoints over 380 seconds. A flight executed from the same vehi- 
cle, on another day and on another route, was utilized to compute the variance $\Sigma_{v}$ of the cruise velocity distribution: $\Sigma_{v} \approx \operatorname{diag}_{3 \times 3}([0.0625,0.0625,0.0289])(\mathrm{m} / \mathrm{s})^{2}$.

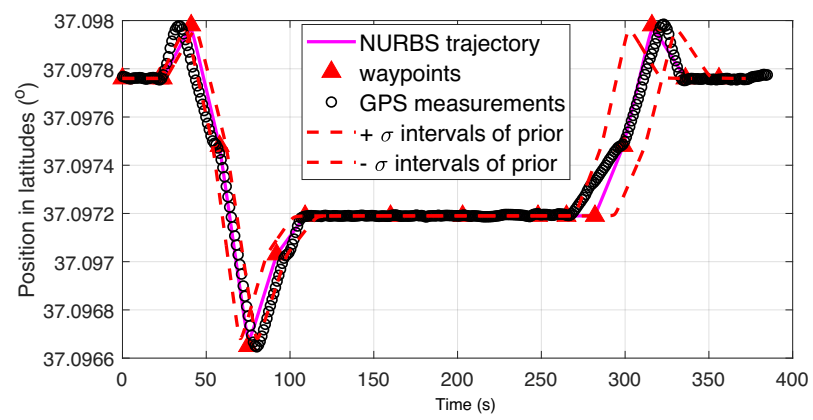

(a)

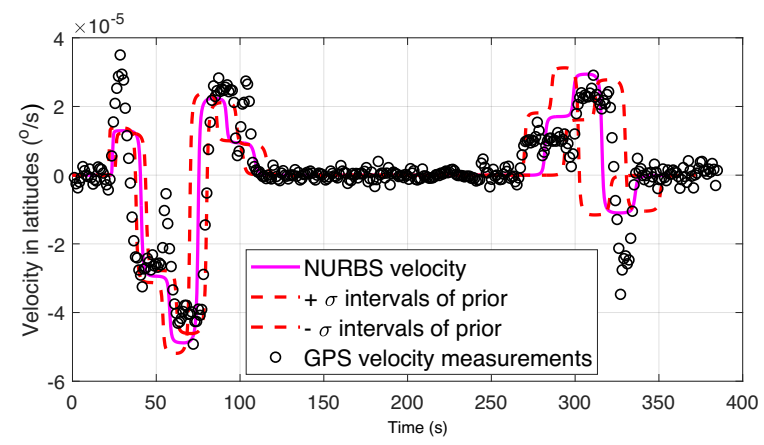

(b)

Figure 3. Experimental UAV flight data with GPS measurements and prior uncertainty bounds for (a) position profile (b) velocity profile.

If wind field is known for the flight path, the wind velocity vector can be added to the velocity uncertainty as well. For example, the notation $\boldsymbol{w}=\left[w_{x}, w_{y}, w_{z}\right]^{T} \in \mathbb{R}^{3 \times 1}$ is used to indicate the wind field components along $\mathrm{x}$ (East), $\mathrm{y}$ (North) and $\mathrm{z}(\mathrm{Up})$ directions, with corresponding uncertainty $\Sigma_{w}=$ $I_{3}\left[\sigma_{w_{x}}^{2}, \sigma_{w_{y}}^{2}, \sigma_{w_{z}}^{2}\right]^{T} \in \mathbb{R}^{3 \times 3}$ for Gaussian pdfs. By adding the wind speed in vector form, the ground speed of the vehicle becomes $\boldsymbol{v}_{g}=\boldsymbol{v}+\boldsymbol{w}$. Leveraging once more the algebra of random variables (Physical Sciences 2, 2013), the error interval on the arrival time, previously defined by (6) can be described as in (9).

$\sigma_{t_{j, k}}^{2} \triangleq\left\{\begin{array}{lll}\Delta t_{a, k}{ }^{2} \frac{\sigma_{v_{j, k}}^{2}+\sigma_{w_{j, k}}^{2}}{\bar{v}_{g, k}^{2}}, & \forall \begin{array}{l}\bar{v}_{g_{j, k}} \in \overline{\boldsymbol{v}}_{g_{k}}: \bar{v}_{g_{j, k}} \neq 0 \\ 0\end{array} \quad \text { otherwise }\end{array}\right.$

Further, measured location from navigation sensors on-board of the UAV can be used to update the current estimates of UAV position as well as compute the remaining future trajectory. Apart from discrepancies in planned velocity, measurement error from the UAV navigation units constitutes an additional source of uncertainty in the predicted trajectory (Banerjee \& Corbetta, 2020).

In order to integrate measurement error in the uncertainty on predicted trajectory, the NURBS trajectory defined in Eq. (2) defines the prior PDF of the position estimates at time $t=0 \mathrm{~s}$ and velocity estimates at time $t=0$ and $t=1 \mathrm{~s}$, denoted in Eq. (12).

$$
\begin{aligned}
& \sigma_{d, t=t_{0}}= \max \left(\mathcal{P}^{+}\left(t=t_{0}\right)-\mathcal{P}\left(t=t_{0}\right),\right. \\
&\left.\mathcal{P}^{-}\left(t=t_{0}\right)-\mathcal{P}\left(t=t_{0}\right)\right) \\
& d_{0}^{p} \sim \mathcal{N}\left(P_{t=t_{0}}, \sigma_{d, t=t_{0}}^{2}\right) \\
& v_{0}^{p} \sim \mathcal{N}\left(V_{t=t_{0}}, \sigma_{v, t=t_{0}}^{2}\right) \\
& v_{1}^{p} \sim \mathcal{N}\left(V_{t=t_{1}}, \sigma_{v, t=t_{1}}^{2}\right)
\end{aligned}
$$

Next, the trajectory profile is converted to a discrete timestate space model such that the position is a function of position at the previous time step and parameters $\boldsymbol{\theta}_{t-1: t}=\left(v_{t-1}, v_{t}^{p}\right)$.

$$
\begin{aligned}
& d_{t}=d_{t-1}^{p}+v_{t-1}^{p} \delta t+\frac{1}{2} a_{t-1} t \delta t^{2} \\
& =d_{t-1}^{p}+v_{t-1}^{p} t+\frac{1}{2}\left(v_{t}^{p}-v_{t-1}^{p}\right) \delta t
\end{aligned}
$$

Finally, the uncertainty bounds on the predicted trajectory is obtained via implementation of Kalman filters (15).

$$
\begin{aligned}
\hat{\boldsymbol{x}}_{k \mid k-1} & =A \hat{\boldsymbol{x}}_{k-1 \mid k-1}+B \boldsymbol{u}_{k-1}+E \boldsymbol{e}_{k-1} \\
P_{k \mid k-1} & =A P_{k-1 \mid k-1} A^{T}+Q
\end{aligned}
$$

where, the state vector $\boldsymbol{x}=\left[d_{x}, d_{y}, d_{z}, v_{x}, v_{y}, v_{z}\right]^{T}$, containing the three positions $d$ and velocities $v$ in the three Cartesian directions. The input vector contain the planned velocity at the next time step, $\boldsymbol{u}_{k-1}=\left[v_{x, k}^{p}, v_{y, k}^{p}, v_{z, k}^{p}\right]^{T}$. A position error term $e$ used to correct any bias that may be introduced by initial conditions or external disturbances is denoted by $\boldsymbol{e}_{k-1}=\left[e_{x, k-1}, e_{y, k-1}, e_{z, k-1}\right]^{T}$.

In this formulation, the state matrix $A$, input matrix $B$, and error-correction matrix $E$ become:

$$
\begin{gathered}
A=0.5\left[\begin{array}{ll}
0_{3} & I_{3} \\
0_{3} & 0_{3}
\end{array}\right] \\
B=0.5\left[\begin{array}{l}
I_{3} \\
0_{3}
\end{array}\right] \\
E=k_{p}\left[\begin{array}{l}
I_{3} \\
0_{3}
\end{array}\right]
\end{gathered}
$$

where $k_{p}$ is a constant analogous to the proportional control 
gain, while $0_{3}$ and $I_{3}$ are an all-zero matrix and the identity matrix, both of dimensions $3 \times 3$. The constant 0.5 in matrices $A$ and $B$ comes from the approximation of uniformlyaccelerated motion where acceleration is computed as the average between speed at two subsequent time steps (see Eq. (14)). Diagonal matrices $P$ and $Q$ of dimensions $6 \times 6$, represent the convariance matrix of the state vector and the model noise. Once the prior of the system state $\hat{\boldsymbol{x}}_{k \mid k-1}$ and its covariance matrix $P_{k \mid k-1}$ have been computed, the updating is performed following the Kalman filter procedure, Eq. (16).

$$
\begin{aligned}
\tilde{\boldsymbol{y}}_{k} & =\boldsymbol{m}_{k}-H \hat{\boldsymbol{x}}_{k \mid k-1} \\
S_{k} & =H P_{k \mid k-1} H^{T}+\Sigma_{\text {meas }} \\
K_{k} & =P_{k \mid k-1} H^{T} S_{k}^{-1} \\
\hat{\boldsymbol{x}}_{k \mid k} & =\hat{\boldsymbol{x}}_{k \mid k-1}+K_{k} \tilde{\boldsymbol{y}}_{k} \\
P_{k \mid k} & =\left(I-K_{k} H\right) P_{k \mid k-1}
\end{aligned}
$$

The three position measurements are collected in vector $\boldsymbol{m}$. Matrix $H$ represents the measurement model while $\Sigma_{\text {meas }}$ represents the covariance matrix of the measures and matrix $K$ represents the Kalman gain.

After computing the posterior estimate of the system state, composed of state vector $\hat{\boldsymbol{x}}_{k \mid k}$ and covariance matrix $P_{k \mid k}$, the prediction of the remaining trajectory involves the propagation of the state vector as stated in Eq. (15).

Figure 4 depicts the $95 \%$ confidence intervals on estimated and predicted position values using measurements available up to (a) 50, (b) 150 and (c) 280 seconds of the flight. As observed from the plots, it is evident that uncertainty bounds become tighter when more measurements are available for prediction. Moreover, the uncertainty is higher at the waypoint locations where the UAV changes its direction yielding higher deviations from its planned velocity. Besides, the difference of measurement vector from planned state and the measurement error is taken into account while computing the uncertainty bounds on the estimated trajectory.

The current trajectory tracking technique does not comprise of subsystem behavior such as individual rotor dynamics. As a result, any anomaly or fault in a specific motor or arm of the multi-copter will not be reflected in the simulated flight. Uncertainty introduced from approximation of UAV flight dynamics will be investigated in future studies.

\subsection{Powertrain Health Diagnosis}

In this section, the predictive process is applied to the design of a model-based diagnostic system for an electrical powertrain typically installed in small, low-cost UAVs. The powertrain system model comprises of an electrochemistry model for Li-ion batteries developed in (Daigle \& Kulkarni, 2013), a model for the electronic speed controller (ESC) developed in (Gorospe, Kulkarni, Hogge, Hsu, \& Ownby, 2017), and

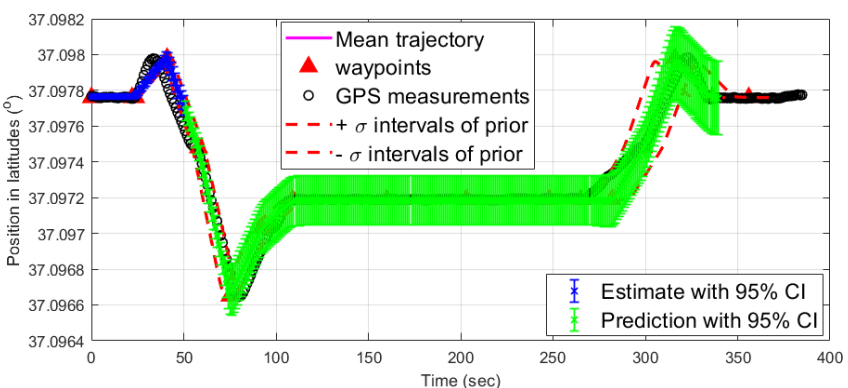

(a)

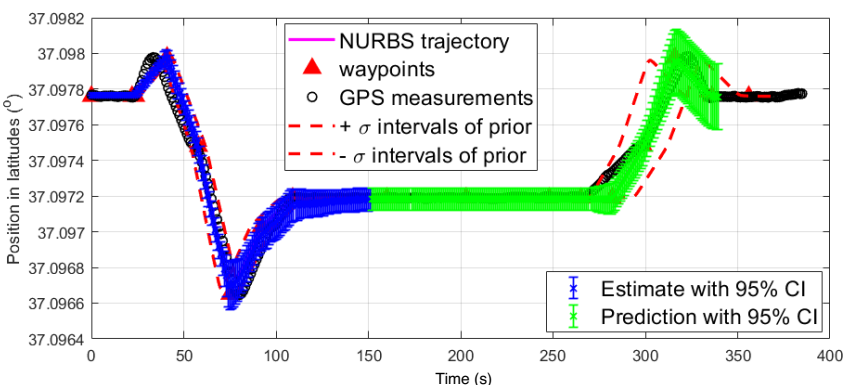

(b)

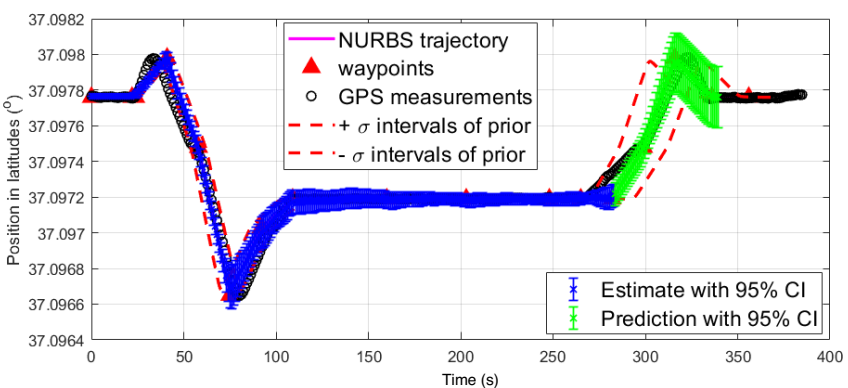

(c)

Figure 4. Trajectory estimation and prediction using Bayes filtering with on-board navigation measurements available upto (a) $50 \mathrm{sec}$ (b) $150 \mathrm{sec}$ and (c) $280 \mathrm{sec}$.

a dynamic model of the brushless DC motor utilized to actuate rotors. Part of this work has already been presented in (Corbetta \& Kulkarni, 2019), and so only a summary is reported here. In this exemplifying discussion, un-modeled physical phenomena are neglected, therefore uncertainty referring to the model abstraction is not considered. Moreover, uncertainty in the battery model parameters is also neglected. The reason is the large number of such parameters, which would require a careful discussion on the parameter modeling strategy, the selection of the distribution functions or the fine tuning of dispersion indices to prevent model instability. Modeling the uncertainty of external disturbances coming from rotors would also require large efforts beyond the scope of this example, and therefore uncertainty from exogenous variables is also neglected. Instead, this Section discusses how to model uncertainty affecting voltage values in the electrochemistry Li-ion battery model, uncertainty rising from lack of a MOSFET model for the ESC, and the effect 
of uncertainty over ESC switch failures. Also, example of uncertain inertia and friction parameters of the DC motor are briefly discussed.

The diagram of the health monitoring system is presented in Figure 5.

\subsubsection{Uncertainty representation in powertrain compo- nent models}

The ESC is modeled as an ideal power inverter employing pulse width modulation (PWM) and half-bridge drivers for each of the three phases within a control block. The PWM modulates three sine waves with carrier frequency $f$ and phase shifts $\varphi=\{0,2 \pi / 3,4 \pi / 3\}$ using a saw-tooth wave with frequency $f_{s t}$. The output is a three phase voltage with duty cycle depending on $f$ and $f_{s t}$, (Pillay \& Krishnan, 1989), (Holtz, 1992).

The model structure composes of two inputs; battery output voltage $V$ and the three modulated square waves from PWM, $F_{1}, F_{2}$, and $F_{3}$. The switch matrix is a design property of the system, and therefore can be considered as a model parameter. The three modulated square waves are pre-multiplied by the switch matrix and the battery's output voltage, as in Eq. (17). The output is a three-phase voltage $v_{a b}, v_{b c}, v_{c a}$, with phase shift of $\pm 2 / 3 \pi$ among one another, which becomes the input of the motor's electrical dynamic model (Gorospe et al., 2017).

$$
\left[\begin{array}{l}
v_{a b} \\
v_{b c} \\
v_{c a}
\end{array}\right]=V\left[\begin{array}{ccc}
1 & -1 & 0 \\
0 & 1 & -1 \\
-1 & 0 & 1
\end{array}\right]\left[\begin{array}{l}
F_{1} \\
F_{2} \\
F_{3}
\end{array}\right]
$$

Even if not specified, battery's output voltage $V$ and output PWM signals $F_{i}, \forall i=\{1,2,3\}$ are obviously time-varying.

Uncertainty affecting each single component is modeled as independent in different ways. The sine wave carrier frequency $f$ can be subject to steady, slow decrease of its value during operation because of the degradation affecting the MOSFETs (Gorospe et al., 2017), which are not modeled in the framework proposed here. Therefore, a possible approach would be to model $f$ as a random variable with small uncertainty around its nominal value. Uncertainty on $f$ should be represented by an always-negative rate of change, to ensure that $f$ is actually decreasing, and not increasing, over time. As already discussed in (Corbetta \& Kulkarni, 2019), a multiplicative, log-Normally distributed random process can achieve the goal,

$$
f_{k}=f_{k-1}-\left.\frac{\mathrm{d} f}{\mathrm{~d} t}\right|_{k-1} e^{\eta}
$$

where the deterministic value $\mathrm{d} f / \mathrm{d} t$ is multiplied by $e^{\eta}$, and $\eta \sim \mathcal{N}\left(-\sigma_{\eta}^{2} / 2, \sigma_{\eta}^{2}\right)$. The function $\mathrm{d} f / \mathrm{d} t$ as well as $\sigma_{\eta}^{2}$ should be tuned based on historical data on MOSFETs degradation.

Switch failures can be modeled by abrupt changes in the switch matrix in (17), using a typical reliability-based approach (Ginart, Brown, Kalgren, \& Roemer, 2009), where switch reliability is defined by failure rates $\lambda(t)$. The elements equal to 1 and -1 in the switch matrix will be reduced to 0 , when a failure of the corresponding MOSFET switches occurs (Celaya, Saxena, Kulkarni, Saha, \& Goebel, 2012). By so doing, uncertainty in the switch matrix is defined through mean-time-betweenfailure or similar quantities. An example of the three-phase voltages from a switch failure is shown in Figure 6, where the element $(2,3)$ of the switch matrix in Eq. (17), originally equal to -1 , has been replaced by 0 .

The structures of the motor model and the battery discharge model are sets of first order differential equations, in the form:

$$
\dot{\boldsymbol{x}}=f_{\boldsymbol{p}}(\boldsymbol{x}, \boldsymbol{u})
$$

where $\boldsymbol{p}$ is the vector of model parameters and $\boldsymbol{u}$ is the input vector. State vectors are defined by

$$
\begin{gathered}
\boldsymbol{x}=\left[q_{s, p}, q_{b, p}, q_{b, n}, q_{s, n}, V_{o}^{\prime}, V_{\eta, p}^{\prime}, V_{\eta, n}^{\prime}\right]^{T}, \\
\boldsymbol{x}=\left[i_{a}, i_{b}, \omega_{m}\right]^{T},
\end{gathered}
$$

for the battery and motor model, respectively. The motor model assumes the form

$$
\dot{\omega}_{m}=\frac{1}{J}\left[-B \omega_{m}+\left(T_{e}-T_{l}\right)\right]
$$

where $T_{e}$ and $T_{l}$ are electrical and mechanical (external) toques, respectively. The battery model is composed of several differential equations and the reader is referred to (Daigle \& Kulkarni, 2013) for further details.

For the motor dynamic model, the vector of model parameters is $\boldsymbol{p}=\left[B, J, R_{s}, L_{M}\right]^{T}$, where $B$ is the friction coefficient, $J$ the motor inertia, $R_{S}$ is the resistance for each phase (assumed identical for the three phases), $L_{M}$ is the difference between self and mutual inductance (also assumed equal for each phase). For more information on the motor model the reader is referred to (Gorospe et al., 2017). For the battery model, the parameter vector is not reported for the sake of brevity (since uncertainty on those parameters is not discussed); the complete set of parameters are derived in (Daigle \& Kulkarni, 2013).

The motor model parameters can be extracted from manufacturer data-sheets or estimated from experimental tests, while the parameters of the electro-chemistry battery model do require estimation from characterization test profiles. The input of the battery model is the applied current $\boldsymbol{u}=i$. The input of the motor model composes of: (i) two of the three-phase input 


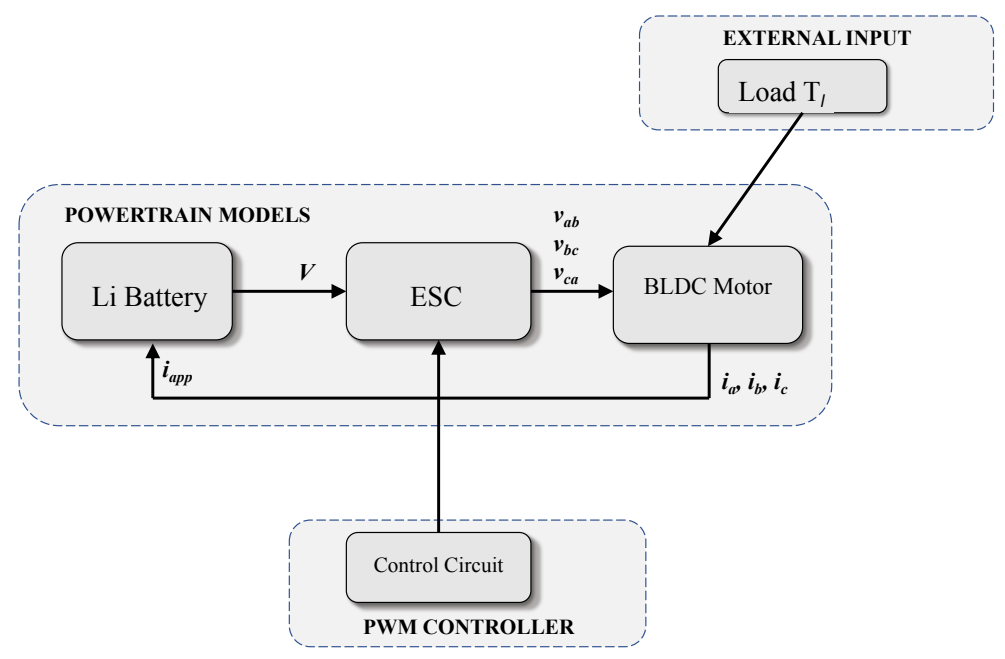

Figure 5. Framework for powertrain health monitoring uncertainty quantification.
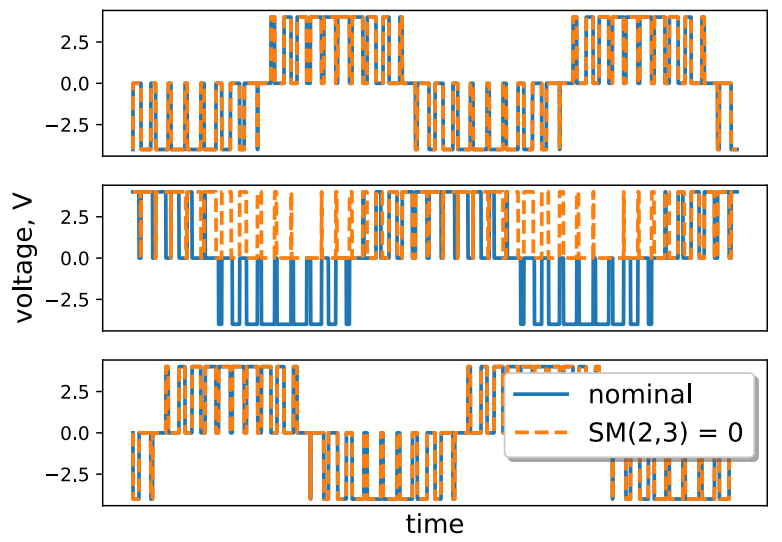

Figure 6. Example of three-phase voltages from ESC with a switch failure. The legend indicates that the switch matrix (SM) element in position $(2,3)$ has been replaced by 0 .

voltages $\left(v_{a b}\right.$ and $\left.v_{b c}\right)$ (ii) three-phase back-emf voltages $e_{a}$, $e_{b}, e_{c}$, and (iii) load torque $T_{l}$, so $\boldsymbol{u}=\left[v_{a b}, v_{b c}, e_{a}, e_{b}, e_{c}, T_{l}\right]^{T}$. It should be noticed that motor model input vector is composed of both operational input, that is the desired applied three-phase voltages, and external input $T_{l}$ which depends on exogenous variables.

Focusing on the battery model, the QoI is the output voltage $V$, which defines the energy introduced in the powertrain to produce the torque on the rotors and thus the thrust to operate the vehicle. Given the complexity of the electro-chemistry battery model, a sampling-based approach appears to be reasonable. The state vector $\boldsymbol{x}$ is considered a random vector, where the Li-ions $q_{s, p}, q_{b, p}$ are defined by random variables. They represent the number of Li-ions on the positive side of the surface $q_{s, p}$ and bulk $q_{b, p}$ of the cell, respectively. Since $q_{s, n}, q_{b, n}$, as well as the voltages $V^{\prime}$, are derived quantities, they also become random variables. Independent, Gaussian pdfs have been utilized to compute random realizations of $q_{s, p}, q_{b, p}$ in a Monte Carlo fashion, using Euler's forward method:

$$
\begin{aligned}
& q_{s, p, k}=q_{s, p, k-1}+\dot{q}_{s, p, k-1} \Delta t_{k-1}+\sigma_{q_{s, p}} \sqrt{\Delta t_{k-1}} r_{1} \\
& q_{b, p, k}=q_{b, p, k-1}+\dot{q}_{b, p, k-1} \Delta t_{k-1}+\sigma_{q_{b, p}} \sqrt{\Delta t_{k-1}} r_{2}
\end{aligned}
$$

where $r_{1}$ and $r_{2}$ are two independent realizations from the standard Normal distribution, and $k$ indicates the time step. Rates of change $\dot{q}_{s, p, k-1}$ and $\dot{q}_{b, p, k-1}$ are derived from the battery dynamic model. The random shocks introduced by $\sigma_{q_{l, p}} r_{1}$ and $\sigma_{q_{l, p}} r_{2}$ are scaled by $\sqrt{\Delta t}$ for consistency with Wiener process and Brownian motion used in stochastic differential equations (Lawler, 2010). By so doing, the variances of the two stochastic processes scale linearly with time. The two standard deviations $\sigma_{q_{s, p}}$ and $\sigma_{q_{b, p}}$ should be properly quantified to reflect the variability observed in experimental tests.

Figure 7 shows, as an example, the output of a single cell modeled using Eq. (19), with initial voltage $V_{0}=V(t=$ $0) \sim \mathcal{N}(4.6,0.316), \sigma_{q_{s, p}}^{2}=\sigma_{q_{b, p}}^{2}=10.0$, and assuming a constant discharge rate with required power $P=8 \mathrm{~W}$. The simulation parameters used are $\Delta t=1 \mathrm{e}-1 \mathrm{~s}, N=1000$ samples, and final simulation time $100 \mathrm{~s}$. The kernel density estimate (KDE) of the voltage at time $100 \mathrm{~s}$, computed with the Monte Carlo samples (MCS) and Gaussian kernel with bandwidth equal to 0.125 , is compared against a Gaussian distribution, Figure 7b. Despite the nonlinearity of the model, the output voltage might be represented by a Gaussian random variable, provided that the propagation of uncer- 


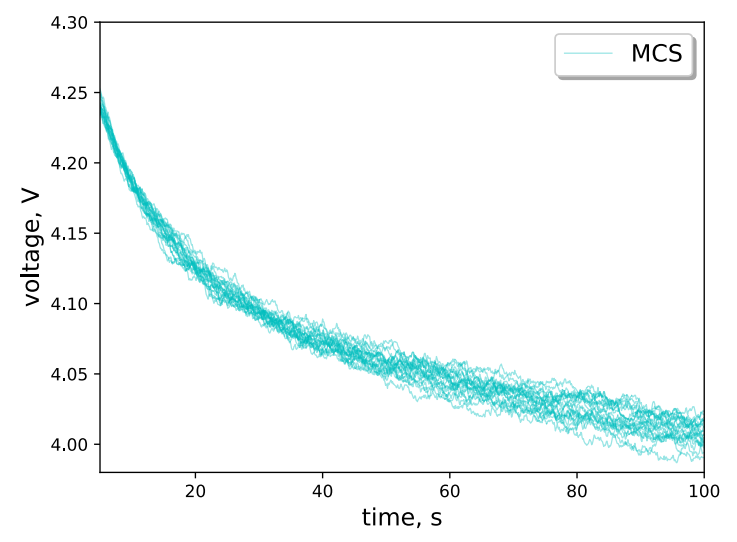

(a)

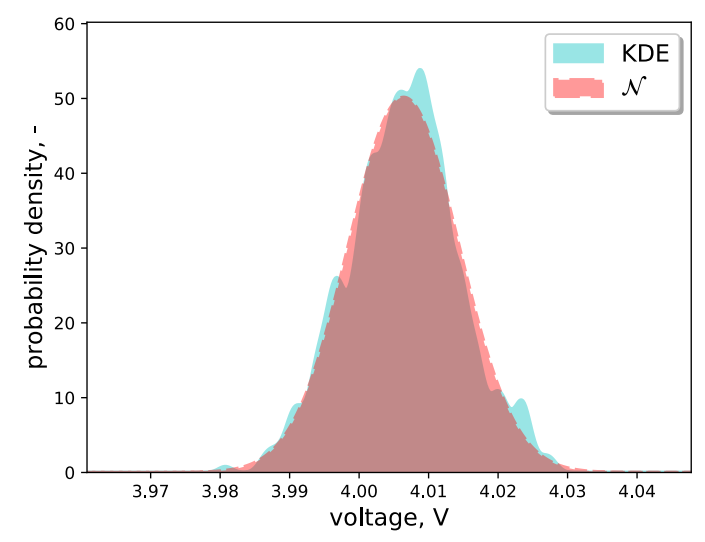

(b)

Figure 7. Simulation of battery discharge at constant power. Monte Carlo samples are shown in $7 \mathrm{a}$, while the resulting kernel density estimate is compared against a Gaussian distribution in $7 \mathrm{~b}$. Only a subset of all samples has been represented in $7 \mathrm{a}$ to appreciate the different paths.

tainty in time can be also be represented by this simplifying assumption.

As clarified in the previous paragraphs, motor model parameters could be represented by random variables to encapsulate the model parameter uncertainty within the model. Sensitivity analyses can aid the selection of important model parameters that should be modeled as random variables, however a few general rules can be applied to all parameters. Because of their physical meaning, all model parameters in $\left[B, J, R_{s}, L_{M}, k_{e}\right]^{T}$ have to be strictly positive, and therefore belonging to $\mathbb{R}^{+}$. As a consequence, Gaussian distributions may not be suitable for describing their uncertainty, especially if their value is (relatively) close to 0 . Let us consider, for example, the friction coefficient $B$ and motor inertia $J$. Their distributions can be defined through a log-Normal transformation by simply using:

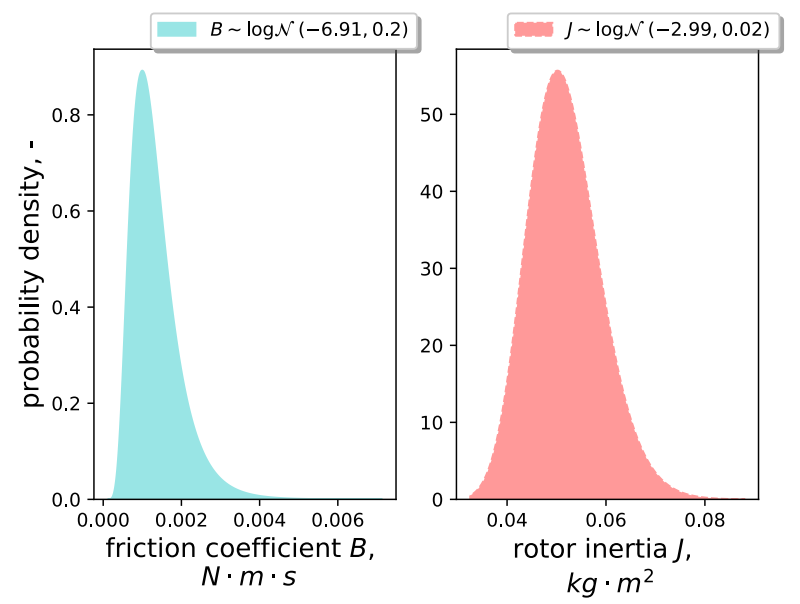

Figure 8. Example of log-Normal probability distributions of $B$ and $J$.

$$
\begin{aligned}
& \ln B \sim \mathcal{N}\left(\mu_{\ln B}, \sigma_{\ln B}^{2}\right), \\
& \ln J \sim \mathcal{N}\left(\mu_{\ln J}, \sigma_{\ln J}^{2}\right)
\end{aligned}
$$

assuming that $B$ and $J$ are independent random variables (the generalization to the case $\sigma_{\ln B, \ln J} \neq 0$ is straightforward by introducing a multi-variate Normal distribution). Examples of the two log-Normal distributions are shown in Figure 8.

An example of angular velocity output from the model in (18) using samples from the distributions of $B$ and $J$ is visible in figure 9. The effect of different inertia values is clearly visible in the transient period necessary to reach the steadystate regime. In order to emphasize the effect of $B$ and $J$ samples, the graph was generated neglecting dynamics effects on the back-emf voltage and external load torque $T_{l}$.

\section{Conclusion}

This paper proposed a framework for uncertainty quantification dedicated to autonomous system tracking and health monitoring. Although being system agnostic, the framework addresses key issues related to automated and autonomous UAVs operations in the airspace.

The developed framework is motivated by the need to ensure a holistic state-awareness of an airspace with multiple vehicles operating within, as expected in the near future for low-altitude airspace in urban and rural environments. Different uncertainty sources that affect the operations of those vehicles must be properly identified and quantified such that look-ahead forecast of the airspace or of vehicle health conditions can highlight airspace as well as vehicle anomalies. To this aim, the proposed approach provides a detailed description of these uncertainty sources classified into model uncer- 


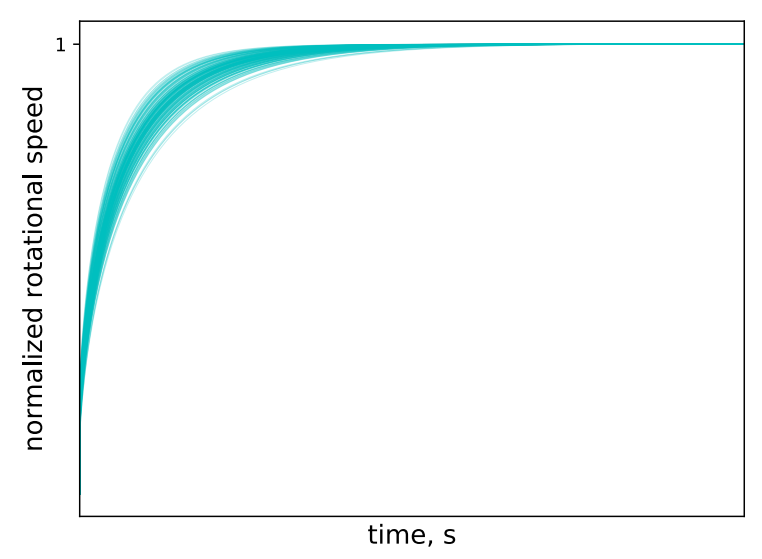

Figure 9. Example of motor speed variations from 0 up to a steady-state regime (nominal speed $\omega_{n}=675$ RPM).

tainty, method uncertainty, measurements uncertainty and input uncertainty. The identified sources are then included and propagated within a predictive process structure composed of different spaces (input space, measure space, representation space and computer space). The methodology helps mapping the relevant uncertainty sources that may enter the prediction process, and define how the identified uncertainty sources interact and impact each other within this predictive process structure.

The proposed approach was demonstrated using two case studies. The first application shows a simple technique to monitor the UAV location and its adherence to the pre-defined flight path. It can provide enough information to compute the expected time of arrival at each location along the path and shrink or enlarge uncertainty bounds of the trajectory profile according to the observed vehicle kinematic profile. The second case study shows how the list of uncertainty sources can aid the design of a vehicle powertrain health monitoring system. According to the granularity of the model-based design, uncertainty can be introduced in different ways, from failure rates coming from reliability analysis to random variables describing physical quantities.

This work does not address a number of topics related to uncertainty quantification: sensitivity analysis of the variables affecting a specific model, statistical techniques to address uncertainty quantification and propagation, as well as methods to describe and interpret uncertainty. It also highlights a few challenges for the effective implementation of monitoring strategies for automated and autonomous vehicles operating in time-varying environments. Characterization of external forcing, like the wind field and the torque acting on the powertrain during flight, is a challenge to be addressed in the future to obtain more meaningful predictions. Such characterization inherently involves uncertainty quantification, given the aleatory nature of some external forcing. This approach shares similarities with system-level prognostics approaches, but introduces further challenges related to the automated or self-directedness properties of the systems. Methods should be capable of resolving (i.e, estimating) and predicting external forcing and environmental factors to enable look-ahead forecast of the system dynamics. This work will benefit from more application studies to highlight further challenges and opportunities to implement effective predictive methods for low-altitude UAV operations.

\section{ACKNOWLEDgments}

This work was supported by the System-Wide Safety (SWS) project under the Airspace Operations and Safety Program within the NASA Aeronautics Research Mission Directorate (ARMD). The authors thank Dr. Andrew Moore and Dr. Patrick Quach from the Dynamic Systems and Control Branch and the Safety Critical Avionics Branch at NASA Langley Research Center, for the flight data utilized in this paper.

\section{REFERENCES}

Abernethy, R., Benedict, R., \& Dowdell, R. (1985). Asme measurement uncertainty.

Adams, B. M., Bohnhoff, W., Dalbey, K., Eddy, J., Eldred, M., Gay, D., ... Swiler, L. P. (2009). Dakota, a multilevel parallel object-oriented framework for design optimization, parameter estimation, uncertainty quantification, and sensitivity analysis: version 5.0 user's manual. Sandia National Laboratories, Tech. Rep. SAND2010-2183.

Alba ker, B., \& Rahim, N. (2009). A survey of collision avoidance approaches for unmanned aerial vehicles. In 2009 international conference for technical postgraduates (techpos) (pp. 1-7).

Arulampalam, M. S., Maskell, S., Gordon, N., \& Clapp, T. (2002). A tutorial on particle filters for online nonlinear/non-gaussian bayesian tracking. Signal Processing, IEEE Transactions on, 50(2), 174-188.

Balaban, E., Roychoudhury, I., Spirkovska, L., Sankararaman, S., Kulkarni, C. S., \& Arnon, T. (2017). Dynamic routing of aircraft in the presence of adverse weather using a pomdp framework. In 17th aiaa aviation technology, integration, and operations conference (p. 3429).

Banerjee, P., \& Corbetta, M. (2020). In-time uav flighttrajectory estimation and tracking using bayesian filters. In 2020 ieee aerospace conference (pp. 1-9).

Cacuci, D. (2003). Sensitivity \& uncertainty analysis: Theory, volume 1. Chapman and Hall/CRC. doi: 10.1201/9780203498798

Celaya, J. R., Saxena, A., Kulkarni, C. S., Saha, S., \& Goebel, K. (2012, Jan). Prognostics approach for power mos- 
fet under thermal-stress aging. In Proceedings annual reliability and maintainability symposium.

Chen, Z. (2003). Bayesian filtering: From kalman filters to particle filters, and beyond. Statistics, 182(1), 1-69.

Corbetta, M., Banerjee, P., Okolo, W., Gorospe, G., \& Luchinsky, D. (2019). Real-time uav trajectory prediction for safety monitoring in low-altitude airspace. In Aiaa aviation forum, uas traffic management iv session.

Corbetta, M., \& Kulkarni, C. S. (2019, September). An approach towards uncertainty quantification and management of unmanned aerial vehicle health. In S. Clements (Ed.), Annual conference of the prognostic and health management society.

Crestaux, T., Ma1 tre, O. L., \& Martinez, J.-M. (2009). Polynomial chaos expansion for sensitivity analysis. Reliability Engineering \& System Safety, 94(7), 1161 - 1172. (Special Issue on Sensitivity Analysis)

Daigle, M., \& Kulkarni, C. (2013, October). Electrochemistry-based battery modeling for prognostics. In Annual conference of the prognostics and health management society 2013 (p. 249-261).

Davis, J. D., \& Chakravorty, S. (2007). Motion planning under uncertainty: application to an unmanned helicopter. Journal of Guidance, Control, and Dynamics, 30(5), 1268-1276.

Drozeski, G. R., Saha, B., \& Vachtsevanos, G. J. (2005). A fault detection and reconfigurable control architecture for unmanned aerial vehicles. In 2005 ieee aerospace conference (pp. 1-9).

Eldred, M., \& Burkardt, J. (2009, Jan). Comparison of nonintrusive polynomial chaos and stochastic collocation methods for uncertainty quantification. 47th AIAA Aerospace Sciences Meeting including The New Horizons Forum and Aerospace Exposition. Retrieved from http://dx.doi.org/10.2514/6.2009-976 doi: 10.2514/6.2009-976

FAA. (2018). Unmanned aerial system (uas) traffic management (utm), concept of operations (Tech. Rep.). Federal Aviation Administration.

Fong, T. (2018). Autonomous systems: Nasa capability overview.

Freeman, P., \& Balas, G. J. (2014). Actuation failure modes and effects analysis for a small uav. In American control conference (acc), 2014 (pp. 1292-1297).

Frew, E., \& Sengupta, R. (2004). Obstacle avoidance with sensor uncertainty for small unmanned aircraft. In $200443 r d$ ieee conference on decision and control (cdc)(ieee cat. no. 04ch37601) (Vol. 1, pp. 614-619).

Ghanem, R. G., \& Spanos, P. D. (1991). Stochastic finite elements: A spectral approach. Springer New York. doi: 10.1007/978-1-4612-3094-6

Ginart, A., Brown, D., Kalgren, P., \& Roemer, M. (2009). Online ringing characterization as a diagnostic tech- nique for igbts in power drives igbts in power drives. In Ieee transactions on instrumentation and measurement (Vol. 58).

Glasheen, K., Pinto, J., Steiner, M., \& Frew, E. W. (2019). Experimental assessment of local weather forecasts for small unmanned aircraft flight. In Aiaa scitech 2019 forum (p. 1193).

Goebel, K. (2017). Prognostics, the science of making prediction. CreateSpace Independent Publishing Platform (1).

Gordon, N. J., Salmond, D. J., \& Smith, A. F. (1993). Novel approach to nonlinear/non-gaussian bayesian state estimation. , 140(2), 107-113.

Gorospe, G. E. J., Kulkarni, C. S., Hogge, E., Hsu, A., \& Ownby, N. (2017). A study of the degradation of electronic speed controllers for brushless dc motors. In Asia pacific conference of the prognostics and health management society 2017.

Haug, A. (2005). A tutorial on bayesian estimation and tracking techniques applicable to nonlinear and nongaussian processes. MITRE Corporation, McLean.

Hoffmann, G., Huang, H., Waslander, S., \& Tomlin, C. (2007). Quadrotor helicopter flight dynamics and control: Theory and experiment. In Aiaa guidance, navigation and control conference and exhibit (p. 6461).

Holtz, J. (1992). Pulsewidth modulation-a survey. IEEE transactions on Industrial Electronics, 39(5), 410420.

Jing, D., \& Haifeng, W. (2013). System health management for unmanned aerial vehicle: conception, state-of-art, framework and challenge. In 2013 ieee 11th international conference on electronic measurement \& instruments (Vol. 2, pp. 859-863).

Johry, A., \& Kapoor, M. (2016). Unmanned aerial vehicle (uav): Fault tolerant design. International Journal of Engineering Technology Science and Research, 3(6), $1-7$.

Jun, M., \& D'Andrea, R. (2003). Path planning for unmanned aerial vehicles in uncertain and adversarial environments. In Cooperative control: models, applications and algorithms (pp. 95-110). Springer.

Kalman, R. E. (1960). A new approach to linear filtering and prediction problems. Journal of basic Engineering, 82(1), 35-45.

Kaminer, I., Pascoal, A., Hallberg, E., \& Silvestre, C. (1998). Trajectory tracking for autonomous vehicles: An integrated approach to guidance and control. Journal of Guidance, Control, and Dynamics, 21(1), 29-38.

King, D. W., Bertapelle, A., \& Moses, C. (2005). Uav failure rate criteria for equivalent level of safety. In International helicopter safety symposium, montreal (Vol. 9).

Kopardekar, P., Rios, J., Prevot, T., Johnson, M., Jung, J., \& Robinson, J. E. (2016). Unmanned aircraft system traffic management (utm) concept of operations. In Aiaa 
aviation forum.

Krish nakumar, K. S., Kopardekar, P. H., Ippolito, C. A., Melton, J., Stepanyan, V., Sankararaman, S., \& Nikaido, B. (2017). Safe autonomous flight environment (safe 50) for the notional last "50 ft" of operation of "55 lb" class of uas. In Aiaa information systemsaiaainfotech@aerospace (p. 0445).

Langelaan, J. W., Alley, N., \& Neidhoefer, J. (2011). Wind field estimation for small unmanned aerial vehicles. Journal of Guidance, Control, and Dynamics, 34(4), 1016-1030.

Lawler, G. F. (2010). Stochastic calculus: An introduction with applications. American Mathematical Society.

Liu, Y., \& Goebel, K. (2018). Information fusion for national airspace system prognostics. In Phm society conference (Vol. 10).

Luttinen, J., \& Ilin, A. (2012, 21-23 Apr). Efficient gaussian process inference for short-scale spatio-temporal modeling. In N. D. Lawrence \& M. Girolami (Eds.), Proceedings of the fifteenth international conference on artificial intelligence and statistics (Vol. 22, pp. 741750). La Palma, Canary Islands: PMLR.

Najm, H. N. (2009, Jan). Uncertainty quantification and polynomial chaos techniques in computational fluid dynamics. Annual Review of Fluid Mechanics, 41(1), 35-52. doi: 10.1146/annurev.fluid.010908.165248

Physical Sciences 2, H. U. (2013). A summary of error propagation. Harvard University Lecture Notes.

Pillay, P., \& Krishnan, R. (1989). Modeling, simulation, and analysis of permanent-magnet motor drives. i. the permanent-magnet synchronous motor drive. IEEE Transactions on industry applications, 25(2), 265273.

Radmanesh, M., Kumar, M., \& Sarim, M. (2018). Grey wolf optimization based sense and avoid algorithm in a bayesian framework for multiple uav path planning in an uncertain environment. Aerospace Science and Technology, 77, 168-179.

Rasmussen, C. E., \& Williams, C. K. (2006). Gaussian processes for machine learning (Vol. 2) (No. 3). MIT Press Cambridge, MA.

Rogers, D. F. (2000). An introduction to nurbs: with historical perspective. Elsevier.

Roy, C. J., \& Oberkampf, W. L. (2011). A comprehensive framework for verification, validation, and uncertainty quantification in scientific computing. Computer methods in applied mechanics and engineering, 200(25-28), 2131-2144.

Saltelli, A., Ratto, M., Andres, T., Campolongo, F., Cariboni, J., Gatelli, D., ... Tarantola, S. (2008). Global sensitivity analysis: the primer. John Wiley \& Sons.

Saltelli, A., Tarantola, S., Campolongo, F., \& Ratto, M. (2004). Sensitivity analysis in practice: a guide to assessing scientific models. Chichester, England.
Sankararaman, S. (2015). Significance, interpretation, and quantification of uncertainty in prognostics and remaining useful life prediction. Mechanical Systems and Signal Processing, 52, 228-247.

Sankararaman, S. (2017). Towards a computational framework for autonomous decision-making in unmanned aerial vehicles. In Aiaa information systems-aiaa infotech@aerospace (p. 0446).

Sankararaman, S., \& Goebel, K. (2015). Uncertainty in prognostics and systems health management. International Journal of Prognostics and Health Management, 6.

Sankararaman, S., Ling, Y., Shantz, C., \& Mahadevan, S. (2009). Uncertainty quantification in fatigue damage prognosis. In Annual conference of the prognostics and health management society (pp. 1-13).

Saxena, A., Celaya, J., Balaban, E., Goebel, K., Saha, B., Saha, S., \& Schwabacher, M. (2008). Metrics for evaluating performance of prognostic techniques. In Prognostics and health management, 2008. phm 2008. international conference on (pp. 1-17).

Smith, R. C. (2013). Uncertainty quantification: theory, implementation, and applications (Vol. 12). Society for Industrial and Applied Mathematics (SIAM).

Stevens, B. L., Lewis, F. L., \& Johnson, E. N. (2015). Aircraft control and simulation: dynamics, controls design, and autonomous systems. John Wiley \& Sons.

Sujit, P., Saripalli, S., \& Sousa, J. B. (2014). Unmanned aerial vehicle path following: A survey and analysis of algorithms for fixed-wing unmanned aerial vehicless. IEEE Control Systems Magazine, 34(1), 42-59.

Tessem, B. (1992). Interval probability propagation. International Journal of Approximate Reasoning, 7(3), 95 - 120. doi: https://doi.org/10.1016/0888613X(92)90006-L

Walker, M. (2010). Next generation prognostics and health management for unmanned aircraft. In 2010 ieee aerospace conference (pp. 1-14).

\section{BIOGRAPHIES}

Matteo Corbetta is a Research Engineer with KBR, at NASA Ames Research Center, Calif. He works at the intersection of engineering, machine learning and uncertainty quantification for autonomous aerospace systems. He received BSc., MSc. and Ph.D. in Mechanical Engineering from Politecnico di Milano, Italy. He is on the Editorial Board of the International Journal of Prognostics and Health Management.

Chetan S. Kulkarni received the B.E. (Bachelor of Engineering) degree in Electronics and Electrical Engineering from University of Pune, India in 2002 and the M.S. and Ph.D. degrees in Electrical Engineering from Vanderbilt University, Nashville, TN, in 2009 and 2013, respectively. His current research interests include physics-based modeling, model-based diagnosis and prognosis for complex systems. Dr. Kulkarni is 
a member of the Prognostics and Health Management (PHM) Society, SM AIAA and the SM IEEE.

Portia Banerjee is a Research Engineer working with KBR at the Prognostic Community of Excellence (PCOE) of NASA Ames Research Center, CA. She completed her Bachelors of Technology degree in Electronics and Communications Engineering from National Institute of Technology Durgapur, India and her Ph.D. in Electrical and Computer Engineering from Michigan State University, USA. Her research interests include statistical signal processing, image processing, data mining, uncertainty management and reliability analysis tailored towards diagnostic and prognostic applications in structures and autonomous systems. She is a member of the AIAA,
IEEE and PHM societies and Executive Committee member of ASME NDE Diagnosis and Prognosis Division.

Elinirina I. Robinson is a Research Engineer with KBR, at NASA Ames Research Center, CA. Prior to joining NASA, she received a Ph.D. in Automatic Control in 2018, jointly from ONERA and CNAM (Paris, France), about model-based prognostics for aerospace systems. She received in 2015 an Engineer's Degree in Electrical Engineering from ENSIP (France) and a MSc. in Automatic Control from University of Poitiers (France). Her research interests include diagnostics and prognostics approaches for electrical propulsion systems, filtering and uncertainty propagation methods based on both stochastic and interval analysis algorithms. 